Forum 2018 · 33:1

https://doi.org/10.1007/s12312-017-0380-1

Online publiziert: 17. Januar 2018

(c) Springer Medizin Verlag GmbH, ein Teil von

Springer Nature 2018

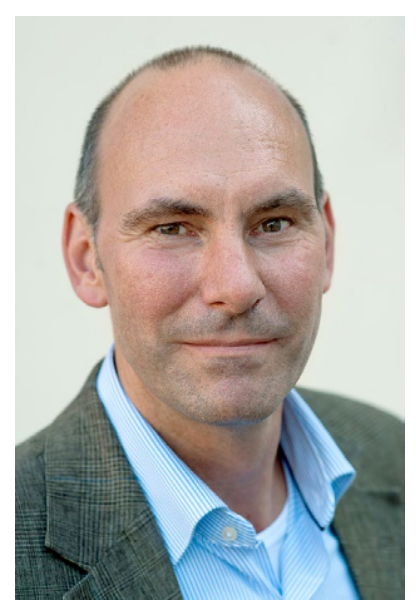

Vom 21. bis zum 24. Februar 2018 findet in Berlin der 33. Deutsche Krebskongress statt. Für die Deutsche Krebsgesellschaft bieten diese 4 Tage die Gelegenheit, Krebs als Erkrankung, als Forschungsgebiet und in seiner ganzen gesellschaftlichen und sozialpolitischen Bedeutung intensiv $\mathrm{zu}$ beleuchten.

Kongresspräsident Professor Thomas Wiegel hat unter dem Motto Krebs verändert Perspektiven ein Programm von großer Spannweite zusammengestellt. Es bildet das ganze Spektrum der Krebsmedizin über alle Phasen der Erkrankung ab, von der qualitativ hochwertigen Diagnosesicherung über die therapeutischen Interventionen bis hin zur verlässlichen und fürsorglichen Begleitung der Betroffenen. Derer, die überleben und derer, die versterben.

Krebsmedizin birgt zahlreiche Kontroversen, gerade auch angesichts des exponentiellen Zuwachses an Wissen und technischen Optionen und der sich wan-

\title{
Krebs verändert Perspektiven
}

delnden politischen, sozialen und regulatorischen Rahmenbedingungen. Um Entwicklung und Fortschritt zuzulassen, sollen Kontroversen - in der Wissenschaft wie im klinischen Alltag - offen diskutiert werden. Der DKK 2018 gibt deshalb der Kontroverse einen bedeutenden Raum. Neben den traditionellen Formaten wie Plenarsitzungen, wissenschaftlichen und edukativen Symposien wurden neu zahlreiche Oxford Debatten in das Programm integriert. Wir freuen uns auf ideenreiche und an mancher Stelle sicher auch leidenschaftlich geführte Diskussionen zu den strittigen Themen der aktuellen Krebsmedizin.

Diese Ausgabe des Forums gibt Einblicke in einige Schwerpunkte des Kongresses. Hans-Peter Schlemmer aus Heidelberg beleuchtet die onkologische Bildgebung im Wandel und wirft damit ein Schlaglicht auf die wachsende Bedeutung einer hochdifferenzierten Diagnostik als Grundlage der Therapieplanung. Zwei Beiträge befassen sich mit der Begleitung sterbender Menschen. Patrick Jahn aus Halle stellt die Rolle der palliativen Pflege dar. Die Krebspreisträgerin Anja Mehnert aus Leipzig und Sigrun Vehling aus Hamburg greifen die psychoonkologische Unterstützung von Patienten und Angehörigen in der Sterbephase auf. Der oftmals tabuisierte Sterbewunsch von Krebspatienten wird von Jan Schildmann (Fürth/München) thematisiert. Der angemessene Umgang damit erfordert neben klinischen auch ethische, rechtliche und kommunikative Kompetenzen. Einen Fokus stellt in seinem Beitrag wie auch auf dem Kon- gress die in der klinischen Praxis kontrovers diskutierte Assistenz zur Selbsttötung dar.

Eine berechtigte Erwartung der $\mathrm{Pa}$ tienten an die Krebsmedizin stellt neben dem Zugänglichmachen von Fortschritt und Innovation auch die Vermeidung von Fehlern und Schaden dar. Christoph Scholz aus Ulm greift das Thema der Schadensvermeidung und des Umgangs mit Fehlern in der Onkologie auf.

Wir freuen uns, Sie, die Mitglieder der Deutschen Krebsgesellschaft, und alle an der Krebsmedizin interessierten Kollegen, Experten, Betroffenen und Akteure im Gesundheitswesen im Februar 2018 in Berlin zu treffen. Informieren Sie sich gerne über die Schwerpunkte des Kongresses auf unserer Webpräsenz www. dkk2018.de. Was wir heute schon versprechen können: Sie werden mit sehr vielen Anregungen und vertiefter Sachkenntnis nach Hause zurückkehren.

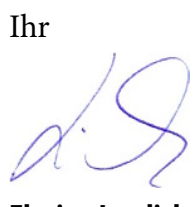

Florian Lordick

\section{Korrespondenzadresse}

Universitäres Krebszentrum Leipzig

(UCCL)

Prof. Dr. med. Florian Lordick

Liebigstraße 20

04103 Leipzig, Deutschland

Tel:0341 9712560

E-Mail: Direktion.UCCL@medizin.uni-leipzig.de 